\title{
Image Processing Based Method for Characterization of the Fat/Meat Ratio and Fat Distribution of Pork and Beef Samples
}

\author{
Jozsef Felfoldi ${ }^{1 *}$, Laszlo Baranyai ${ }^{1}$, Ferenc Firtha ${ }^{1}$, \\ Laszlo Friedrich ${ }^{2}$ and Csaba Balla ${ }^{2}$ \\ ${ }^{1}$ Department of Physics and Control, Corvinus University of Budapest \\ 14-16 Somloi str, Budapest, $\mathrm{H}$-1118 Hungary \\ ${ }^{2}$ Dept. of Refrigeration and Livestocks' Products Technology, Corvinus University of \\ Budapest, 44 Menesi, Budapest, H-1118 Hungary \\ ${ }^{*}$ Corresponding author. E-mail: jozsef.felfoldi@uni-corvinus.hu
}

\begin{abstract}
The fat content (fat distribution) of the pork and beef raw material is one of their most important quality characteristics. Image processing methods were applied to provide with quantitative parameters related to these properties. Different hardware tools were tested to select the appropriate imaging alternative. Statistical analysis of the RGB data was performed in order to find appropriate classification function for segmentation. Discriminant analysis of the RGB data of selected image regions (fat-meat-background) resulted in a good segmentation of the fat regions. Classification function was applied on the RGB images of the samples to identify and measure the regions in question. The fat-meat ratio and textural parameters (entropy, contrast etc.) were determined. Comparison of the image parameters with the sensory evaluation results resulted in encouraging correlation.
\end{abstract}

Key words: meat quality, marbling, image processing.

\section{Introduction}

One of the most important quality characteristics of the pork and beef raw material is related to their fat content. The fat/meat ratio, or the distribution of the fat content (the marbling) are deciding factors in the market value of the samples. There is a definite need in the meat industry and market chains for quick, objective and reliable methods, able to measure parameters, connected to these quality properties. Different research groups applied different approaches to manage this problem. Shiranita et al. (2000) segmented greyscale images by neural network. Jackman and Sun (2009) combined thresholding and clustering to improve segmentation. In some publications, the marbling structure was analysed as well (Taraichi et al, 2002, Hussein, 2011)). Barbin and Sun (2011) call the attention on the advantages of extending the wavelength range of the experiments to NIR.

Our objective was to test the imaging alternatives in different wavelength ranges and to develop methods and software providing with quantitative parameters, comparable with standard meat quality measures.

\section{Material and Methods}

In preliminary experiments 6 to 12 slices of different types of pork and beef samples were used to test the applied recording and image processing methods. In quality evaluation tests 150-150 slice of rib and sirloin samples were qualified by experts into the regular quality categories. After the sensory evaluation, the recorded images of the samples were given to the image processing team for development of classification method.

Different approaches were applied for the image recording: 
2.1. High resolution industrial B\&W camera (MV1-D1312(I) Gigabit Ethernet Series, CMOS Area Scan Camera) was used with multispectral illumination system. The applied light source alternatives were as follows

- White: $\quad 4$ pcs $1 \mathrm{~W}$ Power LED

- Red: $\quad 625 \mathrm{~nm}+/-10 \mathrm{~nm}$ (4 pcs 1 W LED)

- Green: $\quad 525 \mathrm{~nm}+/-12 \mathrm{~nm}$ (4 pcs 1 W LED)

- Blue: $\quad 470 \mathrm{~nm}+/-12 \mathrm{~nm}$ (4 pcs 1 W LED)

- InfraRed: $\quad 850 \mathrm{~nm}+/-20 \mathrm{~nm}$ (4 pcs $70 \mathrm{~mW} / \mathrm{sr}$ infrared power LED)

The multispectral images were recorded in the same position of the samples, this way the records of different wavelength ranges can be investigated in arbitrary combination as well. Images of high dynamic range (12 bit) and $1024^{\star} 1024$ pixel spatial resolution were recorded and stored as lossless BIN files.

2.2. Hyperspectral system: Headwall Photonic Hyperspec ${ }^{T M}$ NIR XS-I320C1-100 imaging spectrometer was used to record meat sample images in 1000-1700 nm range. The pushbroom system recorded the projected line of the sample with 320 pixel spatial resolution and scanned the whole surface of the sample by moving the sample-holder table by $0.5 \mathrm{~mm}$ steps. The records were stored in hypercubes, containing the spatial and spectral information.

2.3. In industrial environment, images of samples (previously qualified by experts) were recorded with a commercial, SLR digital camera (CANON EOS 450D). In order of the reproducibility, the camera was applied with fixed recording parameters (shutter time, aperture and white balance). Special measurement setup with diffuse, homogenous illumination was used to avoid the disturbing effects of the shiny or shadowed details.

The images were processed in Mathcad (V11.0) and with a special program developed in Borland $\mathrm{C}++$ for this purpose. PASW Statistics (18.0.0) software was used for statistical analysis.

\section{Results and Conclusions}

3.1. The multispectral system resulted in four-channel images of the samples. In case of a rib sample, the results are illustrated on Figure 1, together with the intensity diagrams of the multispectral rib images at a marked image-row.

According to the analysis of the image records, we can conclude, that the best meat-to-fat contrast (conclusively the best signal-to-noise ratio, resulting in the most effective segmentation) can be achieved applying the blue $(470 \mathrm{~nm})$ or the green $(525 \mathrm{~nm})$ light sources. This conclusion was confirmed by the shown intensity diagrams. These intensity ratios provide with sufficient information for the further image processing steps, the tested camera with the appropriate illumination (monochromatic blue or green light source) was found to be technically suitable for the image recording. However, the main advantage of this system of relatively high cost (very high dynamic range) is not really needed for processing of the tested meat sample images (the natural variability of the intensity of a meat surface even within a practically homogenous picture detail - is far above the camera noise).

3.2. The hyperspectral system resulted in hypercubes, containing the spatial and spectral information about the tested sample. The spatial projection of a sirloin sample at the wavelength, selected by statistical analysis in order to product effective fat/meat segmentation and the result of the segmentation are shown on FIGURE 2.

Summarizing the results of the tests with hyperspectral system, we can conclude, that the hyperspectral measurements in the $1000-1700 \mathrm{~nm}$ range resulted in no really significant wavelength, enhancing the fat-meat discrimination power. Furthermore, the spatial resolution 
of the applied instrumentation was at the edge of the technical applicability (approx. 0.5 $\mathrm{mm} /$ pixel in case of inspection of a whole slice). Conclusively, the hyperspectral system was excluded from the further investigations.

3.3. Good signal levels of the green and blue channel in 3.1. were found to be encouraging from the point of view of applying an RGB color camera, as an alternative image recording tool. For individual samples of either the beef or the pork samples, the simple segmentation methods, applied on given transformed channels of the RGB images (namely the BLUE, CYAN or SATURATION transformations) resulted in acceptable, but specific segmentation of the fat and meat regions. To achieve a more general, flexible method, suitable for processing of wide range of sample types, it was necessary to develop more general algorithm and software for the segmentation task.

The method for effective segmentation of the fat and meat fractions of an arbitrary type meat sample was based on an interactive teaching process:

- The first step was the interactive selection of characteristic image regions to collect RGB database of the fractions to segment:

- meat fraction

- fat fraction

- background area

- shiny spots

- Statistical analysis of the RGB data was performed in order to find appropriate classification function for segmentation. Discriminant analysis of the RGB data of selected image regions (fat-meat-background) resulted in a good segmentation of the fat regions (Figure 3).

- Coefficients of the Fischer classification function were determined

- Classification function was applied on the RGB images of the samples to identify and measure the regions is question (FIGURE 4 and 5).

In case of the samples, qualified by experts, the Fischer classification function was used to measure the fat-meat ratio, and to produce the binary images of the samples, representing of the fat distribution. These images can be the base of the further work on identification and characterization of the fibre-structure of this fat content.

As an other approach to characterize the pattern structure of the samples, the images were transformed to reach the maximum fat/meat contrast, and the textural parameters (entropy, energy, contrast etc.) were determined. Comparing these pattern characteristics to the sensory evaluation results, high ranking power was found in case of the contrast and the entropy parameters both for pork and for beef samples. Classifying the samples according to these texture parameters (into 0-1-2-3 quality classes) and comparing the ranks to the results of the experts, very high Spearman-correlation was found (above 0.99), offering encouraging possibility of applying the methods in the everyday meat evaluation practice.

\section{Acknowledgements:}

This work was supported by TÁMOP -4.2.1.B-09/1 and TÁMOP-4.2.2/B-10 projects. 


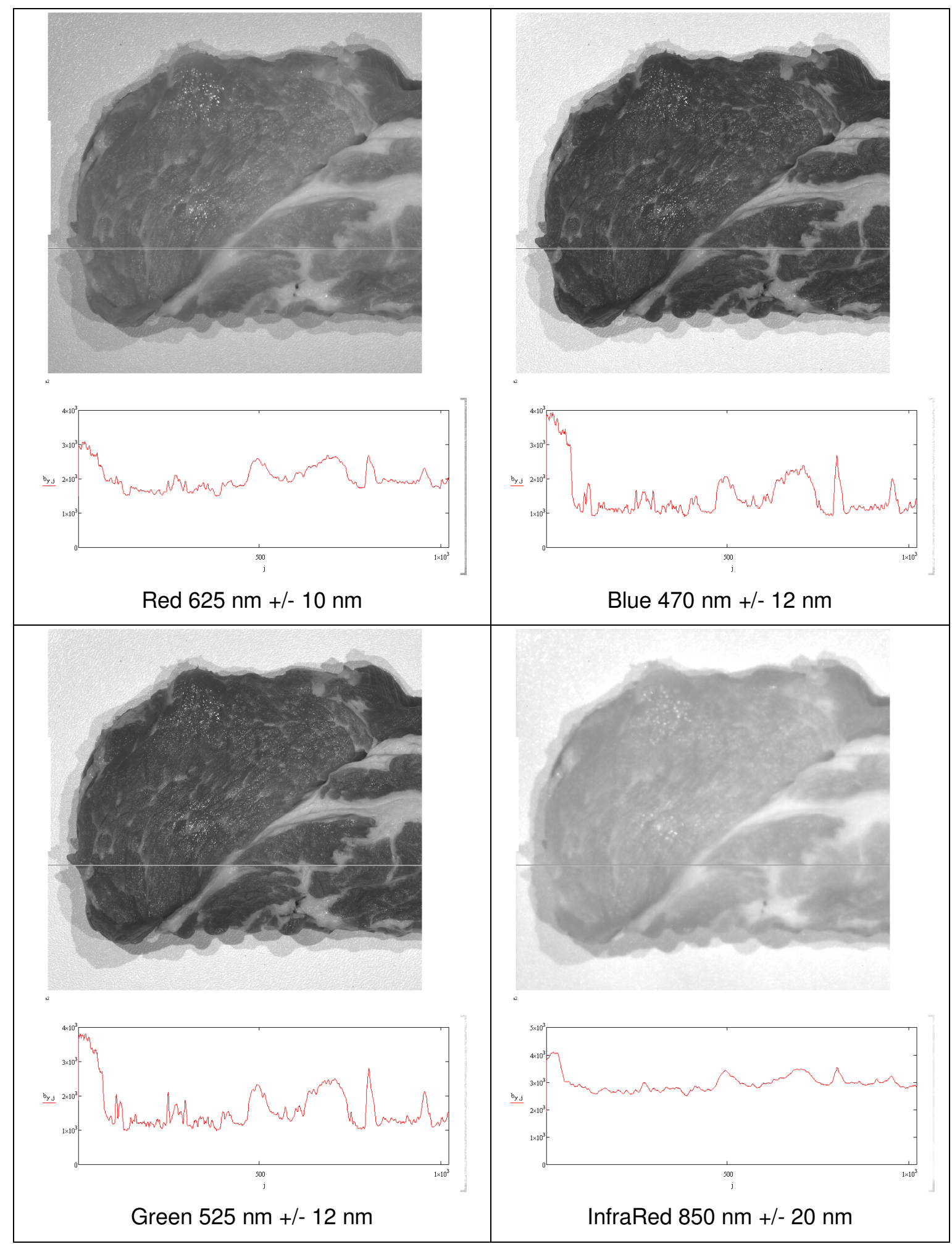

FIGURE 1: High resolution image of a rib sample recorded applying quasi-monochromatic illumination alternatives (LED modules) with the corresponding intensity diagrams of the multispectral rib images at the marked image-row 

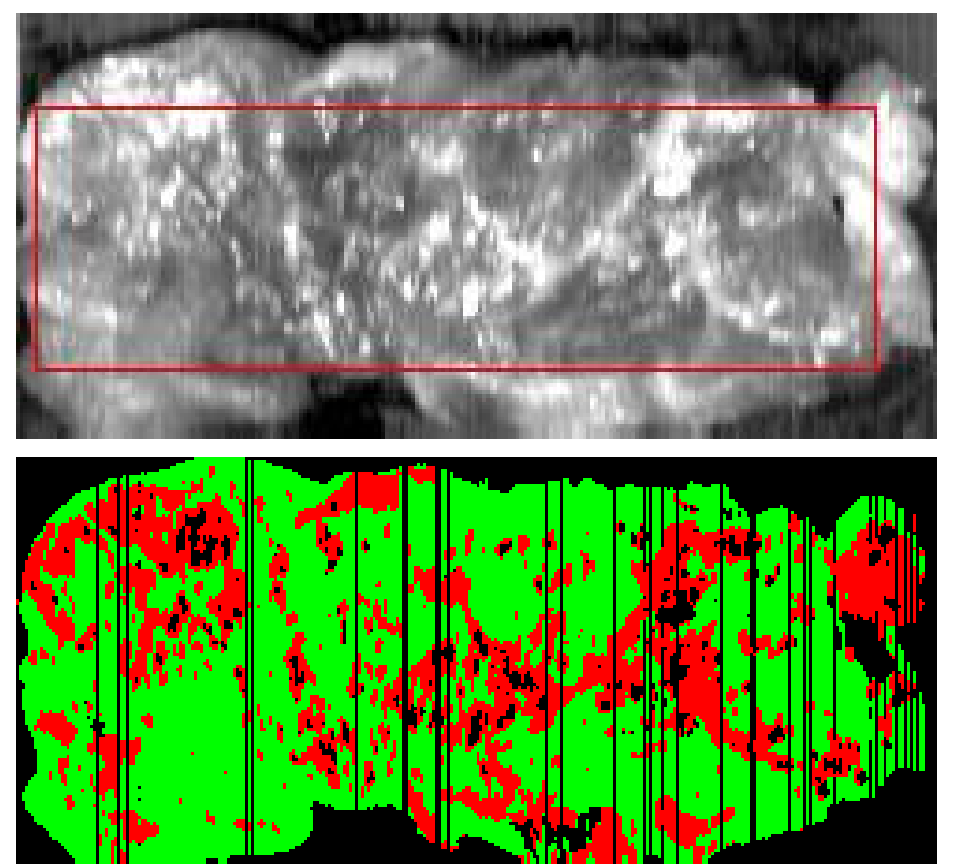

FIGURE 2: Spatial projection of the hypercube of a sirloin sample at a given wavelength and the result of the fat/meat segmentation

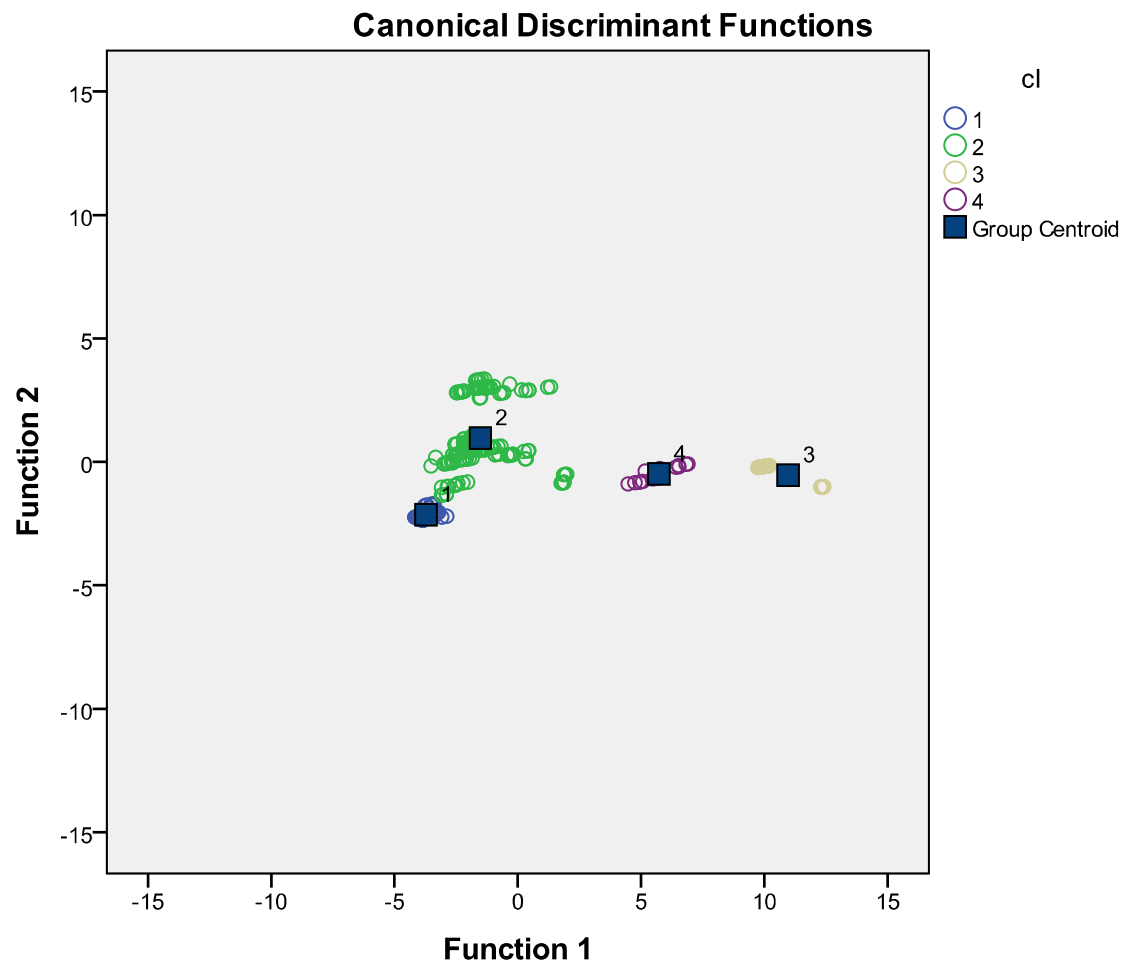

FIGURE 3: Result of the Discriminant Analysis (DA) of the teaching RGB data base (classes: 1: meat, 2: fat, 3: background, 4: shiny spots) 


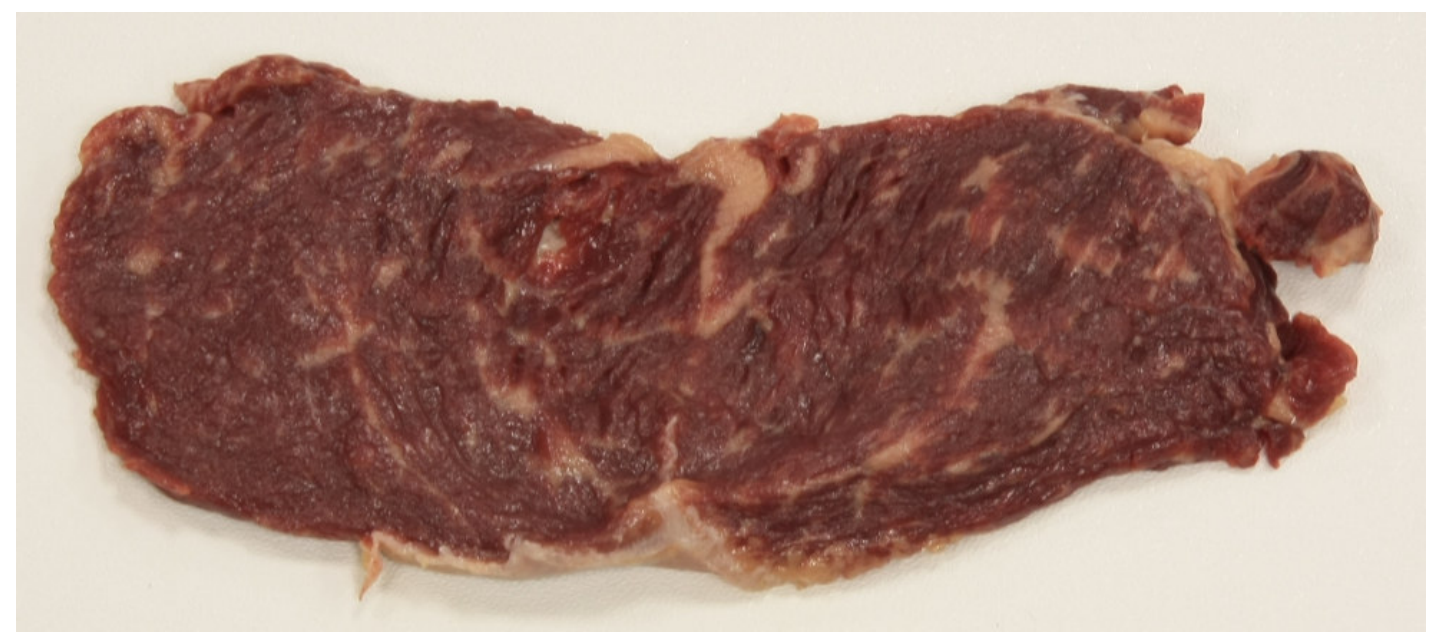

FIGURE 4: Original image of a sirloin sample

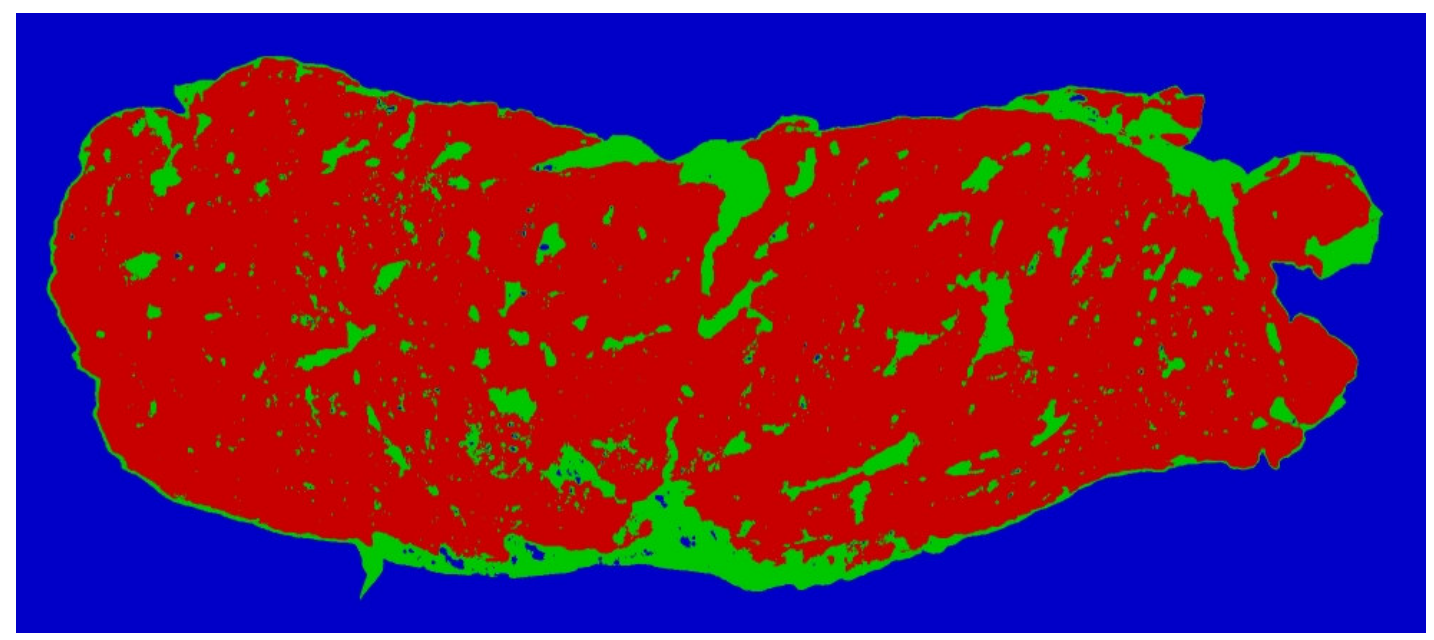

FIGURE 5: Application of the classification function of the Discriminant Analysis on the sample image: (blue: background and shiny spots, red: meat fraction, green: fat fraction)

\section{References}

D. Barbin, G. Elmasry, D. Sun, P. Allen (2012) Near-infrared hyperspectral imaging for grading and classification of pork. Meat Science 90 (2012) 259-268

W.B. Hussein, A.A.Moaty, M.A.Hussein, T.Becker (2011) A novel edge detection method with application to the fat content prediction in marbled meat. Pattern Recognition44(2011)2959-2970

P. Jackman, D. Sun and P. Allen (2009) Automatic segmentation of beef longissimus dorsi muscle and marbling by an adaptable algorithm. Meat Science 83 (2009) 187-194

K. Shiranita, K. Hayashi, A. Otsubo, T. Miyajima and R. Takiyama (2000) Grading meat quality by image processing. Pattern Recognition 33 (2000) 97-104

K. Toraichi, P. W. H. Kwan, K. Katagishi, T. Sugiyama, K. Wada, M. Mitsumoto, H. Nakai, F. Yoshikawa (2002) On a Fluency Image Coding System for Beef Marbling Evaluation. Pattern Recognition Letters 23 (2002) 1277-1291 